\title{
Efficient computing of $n$-dimensional simultaneous Diophantine approximation problems
}

\author{
Attila KOVÁCS \\ Eötvös Loránd University \\ Faculty of Informatics \\ email: \\ attila.kovacs@compalg.inf .elte.hu
}

\author{
Norbert TIHANYI \\ Eötvös Loránd University \\ Faculty of Informatics \\ email: \\ ntihanyi@compalg.inf .elte.hu
}

\begin{abstract}
In this paper we consider two algorithmic problems of simultaneous Diophantine approximations. The first algorithm produces a full solution set for approximating an irrational number with rationals with common denominators from a given interval. The second one aims at finding as many simultaneous solutions as possible in a given time unit. All the presented algorithms are implemented, tested and the PariGP version made publicly available.
\end{abstract}

\section{Introduction}

\subsection{The problem statement}

Rational approximation, or alternatively, Diophantine approximation is very important in many fields of mathematics and computer science. Archimedes approximated the irrational number $\pi$ with 22/7. Long before Archimedes, ancient astronomers in Egypt, Babylonia, India and China used rational approximations. While the work of John Wallis (1616-1703) and Christiaan Huygens (1629-1695) established the field of continued fractions, it began to blossom

Computing Classification System 1998: G.2.0

Mathematics Subject Classification 2010: 68R01, 11J68

Key words and phrases: Diophantine approximation 
when Leonhard Euler (1707-1783), Johann Heinrich Lambert (1728-1777) and Joseph Louis Lagrange (1736-1813) embraced the topic. In the 1840s, Joseph Liouville (1809-1882) obtained an important result on general algebraic numbers: if $\alpha$ is an irrational algebraic number of degree $n>0$ over the rational numbers, then there exists a constant $c(\alpha)>0$ such that

$$
\left|\alpha-\frac{p}{q}\right|>\frac{c(\alpha)}{q^{n}}
$$

holds for all integers $p$ and $q>0$. This result allowed him to produce the first proven examples of transcendental numbers. In 1891 Adolf Hurwitz (1859-1919) proved that for each irrational $\alpha$ infinitely many pairs $(p, q)$ of integers satisfy

$$
\left|\alpha-\frac{p}{q}\right|<\frac{1}{q^{2} \sqrt{5}}
$$

but there are some irrational numbers $\beta$ for which at most finitely many pairs satisfy

$$
\left|\beta-\frac{p}{q}\right|<\frac{1}{q^{2+\gamma} \sqrt{5+\mu}}
$$

no matter how small the positive increments $\gamma$ and $\mu$ are.

The idea can be generalized to simultaneous approximation. Simultaneous diophantine approximation originally means that for given real numbers $\alpha_{1}, \alpha_{2}, \ldots, \alpha_{n}$ find $p_{1}, p_{2}, \ldots, p_{n}, q \in \mathbb{Z}$ such that

$$
\left|\alpha_{i}-\frac{p_{i}}{q}\right|
$$

is "small" for all $i$, and $q$ is "not too large".

For a given real $\alpha$ let us denote the nearest integer distance function by $\|$.$\| ,$ that is, $\|\alpha\|=\min \{|\alpha-\mathfrak{j}|, \mathfrak{j} \in \mathbb{Z}\}$. Then, simultaneous approximation can be interpreted as minimizing

$$
\max \left\{\left\|q \alpha_{1}\right\|, \ldots,\left\|q \alpha_{n}\right\|\right\} .
$$

In 1842 Peter Gustav L. Dirichlet (1805-1859) showed that there exist simultaneous Diophantine approximations with absolute error bound $q^{-(1+1 / n)}$. To be more precise, he showed that there are infinitely many approximations satisfying

$$
\left|q \cdot \alpha_{i}-p_{i}\right|<\frac{1}{q^{1 / n}}
$$


for all $1 \leq i \leq n$. Unfortunately, no polynomial algorithm is known for the simultaneous Diophantine approximation problem. However, due to the $\mathrm{L}^{3}$ algorithm of Lenstra, Lenstra and Lovász, if $\alpha_{1}, \alpha_{2}, \ldots, \alpha_{n}$ are irrationals and $0<\varepsilon<1$ then there is a polynomial time algorithm to compute integers $p_{1}, p_{2}, \ldots, p_{n}, q \in \mathbb{Z}$ such that

$$
1 \leq \mathrm{q} \leq 2^{\mathfrak{n}(\mathrm{n}+1) / 4} \varepsilon^{-n} \text { and }\left|\mathrm{q} \cdot \alpha_{i}-p_{i}\right|<\varepsilon
$$

for all $1 \leq i \leq n$ (see [10]).

Lagarias $[7,8]$ presented many results concerning the best simultaneous approximations. Szekeres and T. Sós [12] analyzed the signatures of the best approximation vectors. Kim et al. [4] discussed rational approximations to pairs of irrational numbers which are linearly independent over the rationals and applications to the theory of dynamical systems. Armknecht et al. [1] used the inhomogeneous simultaneous approximation problem for designing cryptographic schemes. Lagarias [9] discussed the computational complexity of Diophantine approximation problems, which, depending on the specification, varies from polynomial-time to $\mathcal{N} \mathcal{P}$-complete. Frank and Tardos [2] developed a general method in combinatorial optimization using simultaneous Diophantine approximations which could transform some polynomial time algorithms into strongly polynomial.

In this paper we focus on two algorithmic problems. Consider the set of irrationals $\gamma=\left\{\alpha_{1}, \alpha_{2}, \ldots, \alpha_{n}\right\}$. Let $\varepsilon>0$ be real and $1 \leq a \leq b$ be natural numbers. Furthermore, let us define the set

$$
\Omega(\Upsilon, \varepsilon, a, b)=\left\{k \in \mathbb{N}: a \leq k \leq b,\left\|k \alpha_{i}\right\|<\varepsilon \text { for all } \alpha_{i} \in \Upsilon\right\} .
$$

For given $\Upsilon, \varepsilon$ and $a, b$

1. determine all the elements of $\Omega(\Upsilon, \varepsilon, a, b)$,

2. determine as many elements of $\Omega(\Upsilon, \varepsilon, a, b)$ as possible in a given time unit

efficiently. We refer to the first problem as the "all-elements simultaneous Diophantine approximation problem". In case of $|\Upsilon|=n \geq 1$ we call it an $n$ dimensional simultaneous approximation. The second problem is referred to as the "approximating as many elements as possible" problem.

\section{Challenges:}

1. Determine all elements of

$$
\Omega\left(\{\sqrt{2}\}, 10^{-17}, 10^{20}, 10^{21}\right) .
$$


2. Determine as many elements of

$$
\Omega\left(\left\{\frac{\log (p)}{\log (2)}, p \text { prime }, 3 \leq p \leq 19\right\}, 10^{-2}, 1,10^{18}\right)
$$

as possible in a given time unit.

\subsection{The continued fraction approach}

It is well-known that continued fractions are one of the most effective tools of rational approximation to a real number. Simple continued fractions are expressions of the form

$$
a_{0}+\frac{1}{a_{1}+\frac{1}{a_{2}+\cdots}}
$$

where $a_{i}$ are integer numbers with $a_{1}, a_{2}, \ldots>0$. It is called finite if it terminates, and infinite otherwise. These continued fractions are usually represented in bracket form $\left[a_{0}, a_{1}, \ldots, a_{m}, \ldots\right]$, i.e.

$$
C_{0}=\left[a_{0}\right]=a_{0}, \quad C_{1}=\left[a_{0}, a_{1}\right]=a_{0}+\frac{1}{a_{1}}, \quad C_{2}=\left[a_{0}, a_{1}, a_{2}\right]=a_{0}+\frac{1}{a_{1}+\frac{1}{a_{2}}}, \ldots
$$

where $C_{m}$ are called convergents. Clearly, the convergents $C_{m}$ represent some rational numbers $p_{m} / q_{m}$. An infinite continued fraction $\left[a_{0}, a_{1}, a_{2}, \ldots\right]$ is called convergent if its sequence of convergents $C_{m}$ converges in the usual sense, i.e. the limit

$$
\alpha=\lim _{m \rightarrow \infty} C_{m}=\lim _{m \rightarrow \infty}\left[a_{0}, a_{1}, \ldots, a_{m}\right]
$$

exists. In this case we say that the continued fraction represents the real number $\alpha$. The simple continued fraction expansion of $\alpha \in \mathbb{R}$ is infinite if and only if $\alpha$ is irrational. The convergents $C_{m}$ are the best rational approximations in the following sense:

Lemma 1 No better rational approximation exists to the irrational number $\alpha$ with smaller denominator than the convergents $C_{m}=p_{m} / q_{m}$.

Example 2 The simple continued fraction approximation for $\sqrt{2}$ is $[1,2,2, \ldots]$, the sequence of the convergents is

$$
1, \frac{3}{2}, \frac{7}{5}, \frac{17}{12}, \frac{41}{29}, \frac{99}{70}, \frac{239}{169}, \frac{577}{408}, \frac{1393}{985}, \frac{3363}{2378}, \frac{8119}{5741}, \ldots
$$


Among all fractions with denominator at most 29 , the fraction $41 / 29$ is the closest to $\sqrt{2}$, among all fractions with denominator at most 70 , the fraction $99 / 70$ is closest to $\sqrt{2}$, and so on.

Every convergent is a best rational approximation, but these are not all of the best rational approximations. Fractions of the form

$$
\frac{p_{m-1}+j p_{m}}{q_{m-1}+j q_{m}}\left(1 \leq j \leq a_{m+2}-1\right),
$$

are called intermediate convergents or semi-convergents. To get every rational approximation between two consecutive $p_{m} / q_{m}$ and $p_{m+1} / q_{m+1}$, we have to calculate the intermediate convergents.

Example 2 (cont.) The missing intermediate convergents of Example 2 are

$$
\frac{4}{3}, \frac{10}{7}, \frac{24}{17}, \frac{58}{41}, \frac{140}{99}, \frac{338}{239}, \frac{816}{577}, \frac{1970}{1393}, \frac{4756}{3363}, \ldots
$$

The approximations $|\alpha-p / q|$ above are also known as "best rational approximations of the first kind". However, sometimes we are interested in the approximations $|\alpha \cdot q-p|$. This is called the approximation of a second kind.

Lemma 3 [3] A rational number $\mathbf{p} / \mathbf{q}$, which is not an integer, is a convergent of a real number $\alpha$ if and only if it is a best approximation of the second kind of $\alpha$.

In 1997 Clark Kimberling proved the following result regarding intermediate convergents [5]:

Lemma 4 The best lower (upper) approximates to a positive irrational number $\alpha$ are the even-indexed (odd-indexed) intermediate convergents.

Example 2 (cont.) In order to generate many integers q that satisfy

$$
\|\mathrm{q} \cdot \sqrt{2}\|<10^{-5}
$$

one can apply the theory of continued fractions, especially convergents. If $\mathbf{q}_{\mathrm{m}}$ is the first integer that satisfies $\left\|\mathrm{q}_{\mathrm{m}} \cdot \sqrt{2}\right\|<10^{-5}$ in the continued fraction expansion of $\sqrt{2}$, then all convergents with denominator larger than $\mathrm{q}_{\mathrm{m}}$ will satisfy equation (5). 
Example 5 Consider Challenge 1 stated in (3). There are only 3 convergents of $\sqrt{2}$ where $10^{20}<\mathrm{q}_{\mathrm{m}}<10^{21}$. They are

$\frac{233806732499933208099}{165326326037771920630}, \frac{564459384575477049359}{399133058537705128729}, \quad \frac{1362725501650887306817}{963592443113182178088}$.

With intermediate convergents we get 2 more solutions. Hence, with the theory of continued fractions we are able to find only 5 appropriate integers. One may ask how many elements are in the set $\Omega$ in (3)?

Hermann Weyl (1855-1955) and Waclaw Sierpiński (1882-1969) proved in 1910 that if $\alpha \in \mathbb{R} \backslash \mathbb{Q}$ then $\alpha, 2 \alpha, 3 \alpha, \ldots(\bmod 1)$ is uniformly distributed on the unit interval. From this theorem it immediately follows that there are approximately $2(b-a) \varepsilon$ appropriate integers in the $[a, b]$ interval. In Challenge 1 we expect $2\left(10^{21}-10^{20}\right) \cdot 10^{-17}=18000( \pm 1)$ integers. This is by several orders of magnitude more than what we were able to obtain by continued fractions.

\subsection{The Lenstra-Lenstra-Lovász approach}

We have seen in the previous section that Challenge 1 is unsolvable with the theory of continued fractions. Challenge 2 is a 7 -dimensional simultaneous approximation problem and is even more beyond the potentials of continued fractions. Although there is not known polynomial-time algorithm that is able to solve the Dirichlet type simultaneous Diophantine approximation problem, there exists an algorithm that can be useful for similar problems. The LenstraLenstra-Lovász basis reduction algorithm $\left(\mathrm{L}^{3}\right)$ is a polynomial-time algorithm that finds a reduced basis in a lattice [10]. The algorithm can be applied to solve simultaneous Diophantine approximation with an extra condition.

Lemma 6 There exists a polynomial-time algorithm for the given irrationals $\alpha_{1}, \alpha_{2}, \ldots, \alpha_{n}$ and $0<\varepsilon<1$ that can compute the integers $p_{1}, \ldots, p_{n}$ and $q$ such that

$$
\left|\alpha_{i}-\frac{p_{i}}{q}\right|<\frac{\varepsilon}{q}
$$

and

$$
0<\mathrm{q} \leq \beta^{\mathrm{n}(\mathrm{n}+1) / 4} \varepsilon^{-n}
$$

hold for all $1 \leq \mathfrak{i} \leq \mathrm{n}$, where $\beta$ is an appropriate reduction parameter.

The extra condition is the bound $0<\mathrm{q} \leq \beta^{\mathrm{n}(\mathrm{n}+1) / 4} \varepsilon^{-n}$. 
In one-dimension the $\mathrm{L}^{3}$ algorithm provides exactly the continued fraction approach discussed in the previous section, hence $\mathrm{L}^{3}$ is not an effective tool for answering Challenge 1 . And what about the multidimensional case like Challenge 2?

Let $\alpha_{1}, \alpha_{2}, \ldots, \alpha_{n}$ be irrational numbers and let us approximate them with rationals admitting an $\varepsilon>0$ error. Let $X=\beta^{n(n+1) / 4} \varepsilon^{-n}$ and let the matrix $A$ be the following:

$$
A=\left[\begin{array}{ccccc}
1 & 0 & 0 & \ldots & 0 \\
\alpha_{1} X & X & 0 & \ldots & 0 \\
\alpha_{2} X & 0 & X & \ldots & 0 \\
\vdots & & & & \vdots \\
\alpha_{n} X & 0 & 0 & \ldots & X
\end{array}\right]
$$

Applying the $\mathrm{L}^{3}$ algorithm for $\mathrm{A}$, the first column of the resulting matrix contains the vector $\left[q, p_{1}, p_{2}, p_{3}, \ldots, p_{n}\right]^{\top}$ which satisfies $(6)$.

Let us see how the $\mathrm{L}^{3}$ algorithm works in dimension 7. Let $\alpha_{i}=\frac{\log \left(\mathfrak{p}_{i+1}\right)}{\log (2)}$ where $p_{i}$ denotes the $i$-th prime for $1 \leq i \leq 7$, and let $\varepsilon=0.01$. We are looking for an integer $\mathrm{q} \leq 2^{14} \cdot 100^{7}$ that satisfies $\left\|\mathrm{q} \cdot \alpha_{i}\right\|<\varepsilon$ for all $i$. Applying the $\mathrm{L}^{3}$ algorithm we got $q=1325886000944418$. It is easy to verify that $\left\|q \alpha_{i}\right\|<0.01$ holds for all $1 \leq i \leq 7$.

The $\mathrm{L}^{3}$ algorithm can also be applied in higher dimensions, however, there are some cases where the algorithm can not be used efficiently. The real drawback of the method for our purposes is that it is inappropriate for finding all or many different solutions $\mathrm{q}$ in an arbitrary interval. We note that sometimes one can find a few more solutions with a different choice of $\beta$ (but not much more).

It can be concluded that the apparatus of the continued fractions and the $\mathrm{L}^{3}$ algorithm is not appropriate for solving Challenge 1 and Challenge 2 problems. In this paper we present new methods that can be used to solve these kinds of problems efficiently. All the algorithms presented in this paper were implemented and tested in PARI/GP 2.5.3 with an extension of GNU MP 5.0.1. The experimenting environment was an Intel $\AA$ Core i5-2450M with Sandy Bridge architecture. The code can be downloaded from the project homepage ${ }^{1}$.

\footnotetext{
${ }^{1}$ http://www.riemann-siegel.com/
} 


\section{Approximation in the one-dimensional case}

\section{1 "All-elements" approximation}

In this section we present how to calculate all the elements of $\Omega(\Upsilon, \varepsilon, a, b)$ where $\Upsilon=\{\alpha\}$.

For a given $\Omega$ let $k:\{1,2, \ldots,|\Omega|\} \rightarrow \Omega$ monotonically increasing, so $k_{i}$ denotes the ith integer in $\Omega$. Let us define the set

$$
\Delta_{\Omega}=\left\{k_{n+1}-k_{n}: 1 \leq n \leq|\Omega|-1\right\} .
$$

The set $\Delta_{\Omega}$ contains all possible step-sizes between two consecutive $k_{i}$ 's.

Theorem $7\left|\Delta_{\Omega}\right| \leq 3$.

Proof. The proof has two parts. In the first step we construct all the possible three elements of $\Delta_{\Omega}$ and in the second step we show that there is no more. For the given irrational $\alpha$ and an arbitrary $m \in \mathbb{N}$ let

$$
\langle m\rangle= \begin{cases}\|\alpha m\| & \text { if } \alpha m-\|\alpha m\| \in \mathbb{N}, \\ -\|\alpha m\| & \text { if } \alpha m+\|\alpha m\| \in \mathbb{N} .\end{cases}
$$

Let us furthermore define the following open intervals:

$$
A=(-2 \varepsilon,-\varepsilon), B=(-\varepsilon, 0), C=(0, \varepsilon), D=(\varepsilon, 2 \varepsilon) .
$$

Let $m_{1}$ be the smallest positive integer that satisfies $\left\langle m_{1}\right\rangle \in C \cup D$, let $m_{2}$ be the the smallest positive integer that satisfies $\left\langle m_{2}\right\rangle \in A \cup B$ and let $m_{3}=m_{1}+m_{2}$.

The first part of the proof is to show that there is always at least one integer $\left(m_{1}, m_{2}\right.$ or $\left.m_{3}\right)$ that adding to an arbitrary $k_{i} \in \Omega$ always produces a new integer $k_{j} \in \Omega$. Clearly, $\left\langle k_{i}\right\rangle \in B \cup C$ for all $k_{i}$. Let us see the following cases:

$$
\begin{aligned}
\left\langle k_{i}\right\rangle & \in B: \\
& \text { If }\left\langle m_{1}\right\rangle \in C,\left\langle m_{2}\right\rangle \in A \cup B \text { then }\left\langle k_{i}+m_{1}\right\rangle \in B \cup C . \\
& \text { If }\left\langle m_{1}\right\rangle \in D,\left\langle m_{2}\right\rangle \in A \text { and }\left\langle m_{1}+m_{2}\right\rangle \in C \text { then }\left\langle k_{i}+\left(m_{1}+m_{2}\right)\right\rangle \in B \cup C . \\
& \text { If }\left\langle m_{1}\right\rangle \in D,\left\langle m_{2}\right\rangle \in A \text { and }\left\langle m_{1}+m_{2}\right\rangle \in B \text { then }\left\langle k_{i}+\left(m_{1}+m_{2}\right)\right\rangle \in A \cup B . \\
& \text { If }\left\langle k_{i}+\left(m_{1}+m_{2}\right)\right\rangle \in A \text { then }\left\langle k_{i}+\left(m_{1}+m_{2}\right)-m_{2}\right\rangle \in B \cup C . \\
& \text { If }\left\langle m_{1}\right\rangle \in D,\left\langle m_{2}\right\rangle \in B \text { and }\left\langle m_{1}+m_{2}\right\rangle \in C \text { then }\left\langle k_{i}+\left(m_{1}+m_{2}\right)\right\rangle \in B \cup C . \\
& \text { If }\left\langle m_{1}\right\rangle \in D,\left\langle m_{2}\right\rangle \in B \text { and }\left\langle m_{1}+m_{2}\right\rangle \in D \text { then }\left\langle k_{i}+\left(m_{1}+m_{2}\right)\right\rangle \in C \cup D . \\
& \text { If }\left\langle k_{i}+\left(m_{1}+m_{2}\right)\right\rangle \in D \text { then }\left\langle k_{i}+\left(m_{1}+m_{2}\right)-m_{1}\right\rangle \in B \cup C .
\end{aligned}
$$


$\left\langle k_{i}\right\rangle \in C:$

If $\left\langle m_{1}\right\rangle \in C \cup D,\left\langle m_{2}\right\rangle \in B$ then $\left\langle k_{i}+m_{2}\right\rangle \in B \cup C$.

If $\left\langle m_{1}\right\rangle \in C,\left\langle m_{2}\right\rangle \in A$ and $\left\langle m_{1}+m_{2}\right\rangle \in B$ then $\left\langle k_{i}+\left(m_{1}+m_{2}\right)\right\rangle \in B \cup C$.

If $\left\langle m_{1}\right\rangle \in C,\left\langle m_{2}\right\rangle \in A$ and $\left\langle m_{1}+m_{2}\right\rangle \in A$ then $\left\langle k_{i}+\left(m_{1}+m_{2}\right)\right\rangle \in A \cup B$.

If $\left\langle k_{i}+\left(m_{1}+m_{2}\right)\right\rangle \in A$ then $\left\langle k_{i}+\left(m_{1}+m_{2}\right)-m_{2}\right\rangle \in B \cup C$.

If $\left\langle m_{1}\right\rangle \in D,\left\langle m_{2}\right\rangle \in A$ and $\left\langle m_{1}+m_{2}\right\rangle \in B$ then $\left\langle k_{i}+\left(m_{1}+m_{2}\right)\right\rangle \in B \cup C$.

If $\left\langle m_{1}\right\rangle \in \mathrm{D},\left\langle\mathrm{m}_{2}\right\rangle \in A$ and $\left\langle\mathrm{m}_{1}+\mathrm{m}_{2}\right\rangle \in \mathrm{C}$ then $\left\langle\mathrm{k}_{\mathrm{i}}+\left(\mathrm{m}_{1}+\mathrm{m}_{2}\right)\right\rangle \in \mathrm{C} \cup \mathrm{D}$. If $\left\langle k_{i}+\left(m_{1}+m_{2}\right)\right\rangle \in D$ then $\left\langle k_{i}+\left(m_{1}+m_{2}\right)-m_{1}\right\rangle \in B \cup C$.

Let now $X=\Delta_{\Omega} \backslash\left\{m_{1}, m_{2}, m_{3}\right\}$. We claim that $X=\varnothing$. Suppose otherwise, and let $j$ be the smallest index with $m=k_{j+1}-k_{j} \in X$. Clearly, $\langle m\rangle \in$ $A \cup B \cup C \cup D$. We can observe as well that for all $m \in \mathbb{N}, k_{i} \in \Omega,\left\langle k_{i}+m\right\rangle \in B \cup C$ implies $\langle m\rangle \in A \cup B \cup C \cup D$. Then it is easy to see that

- $j>1$, and $k_{i}$ 's are integer linear combinations of $m_{1}$ and $m_{2}$ for all $i \leq j$,

- $m_{1}, m_{2}<m<m_{1}+m_{2}$,

- $\langle m\rangle \in A \cup D$.

If $\langle m\rangle \in A$ then $\left\langle m-m_{2}\right\rangle \in B \cup C$, which contradicts the mimimality of $j$. In the same way, if $\langle m\rangle \in D$ then $\left\langle m-m_{1}\right\rangle \in B \cup C$, which is a contradiction again. Hence, such an $m$ does not exist. The proof is complete.

Finding the integers $m_{1}$ and $m_{2}$ can be done very effectively with the theory of intermediate convergents. It was already discussed that intermediate convergents of an irrational $\alpha$ always produce the best upper and lower approximations to $\alpha$, so $\mathrm{m}_{1}$ and $\mathrm{m}_{2}$ must be intermediate convergents.

Example 5 (cont.) Applying the FindMMM algorithm (Algorithm 1) we have the values

$\mathrm{m}_{1}=59341817924539925$,

$\mathrm{m}_{2}=24580185800219268$,

$\mathrm{m}_{3}=83922003724759193$.

After the precalculation of $\mathrm{m}_{1}$ and $\mathrm{m}_{2}$ it is very easy to compute every $\mathrm{k}_{\mathrm{i}}$ between $10^{20}$ and $10^{21}$. First we have to find an intermediate convergent between $10^{20}$ and $10^{21}$. It can be done in polynomial time with the theory of continued fractions (e.g: 233806732499933208099 ). After that we can add, subtract $\mathrm{m}_{1}$, $\mathrm{m}_{2}$ or $\mathrm{m}_{3}$ until we reach the bounds of the interval. The Weyl equidistribution theorem predicts 18000 integers that solve (3). Applying Challenge 1 Solver 
algorithm (Algorithm 2) we found exactly 18000 integers. The precalculation and the computation of all $\mathrm{k}_{\mathrm{i}}$ values took only $31 \mathrm{~ms}$.

\section{Algorithm 1 FindMMM \\ Description:}

The algorithm is based on Theorem 7 . The algorithm finds the smallest $m_{1}, m_{2}$ and $m_{3}$ integers such that $0<\left\langle m_{1}\right\rangle<2 \varepsilon,-2 \varepsilon<\left\langle m_{2}\right\rangle<0$. The output of the algorithm is $\Delta_{\Omega}=\left\{m_{1}, m_{2}, m_{1}+m_{2}\right\}$. The main while loop in this algorithm (from line 5 to 15) goes through all intermediate convergents to find $m_{1}$ and $m_{2}$. The theory of intermediate convergents ensures that $m_{1}, m_{2} \in q_{i}$ where $q_{i}$ is the $i^{\text {th }}$ intermediate convergent. When $m_{1}$ and $m_{2}$ are found the while loop terminates and the algorithm returns $m_{1}, m_{2}$ and $m_{1}+m_{2}$ in ascending order.

Precondition: $\alpha \in \mathbb{R} \backslash \mathbb{Q}, \alpha>\varepsilon>0$.

1: procedure $\operatorname{FindMMM}(\alpha, \varepsilon)$

2: $\quad i \leftarrow 0$

3: $\quad \mathrm{m}_{1} \leftarrow 0$

4: $\quad \mathrm{m}_{2} \leftarrow 0$

5: $\quad$ while $m_{1}=0$ or $m_{2}=0$ do

6: $\quad i \leftarrow i+1$

7: $\quad \mathrm{q}_{i} \leftarrow i^{\text {th }}$ intermediate convergents of $\alpha$

8: $\quad k \leftarrow \operatorname{FrAC}\left(q_{i} \cdot \alpha\right)$

$\triangleright$ Fractional part of $\mathrm{q}_{i} \cdot \alpha$

9: $\quad$ if $m_{1}=0$ and $k<2 \varepsilon$ then

10: $\quad m_{1} \leftarrow q_{i}$

11: $\quad$ end if

12: $\quad$ if $m_{2}=0$ and $k>1-2 \varepsilon$ then

13: $\quad \mathrm{m}_{2} \leftarrow \mathrm{q}_{\mathrm{i}}$

14: $\quad$ end if

15: end while

16: $\quad \operatorname{RETURN}\left(\min \left(m_{1}, m_{2}\right), \max \left(m_{1}, m_{2}\right), m_{1}+m_{2}\right)$

17: end procedure 


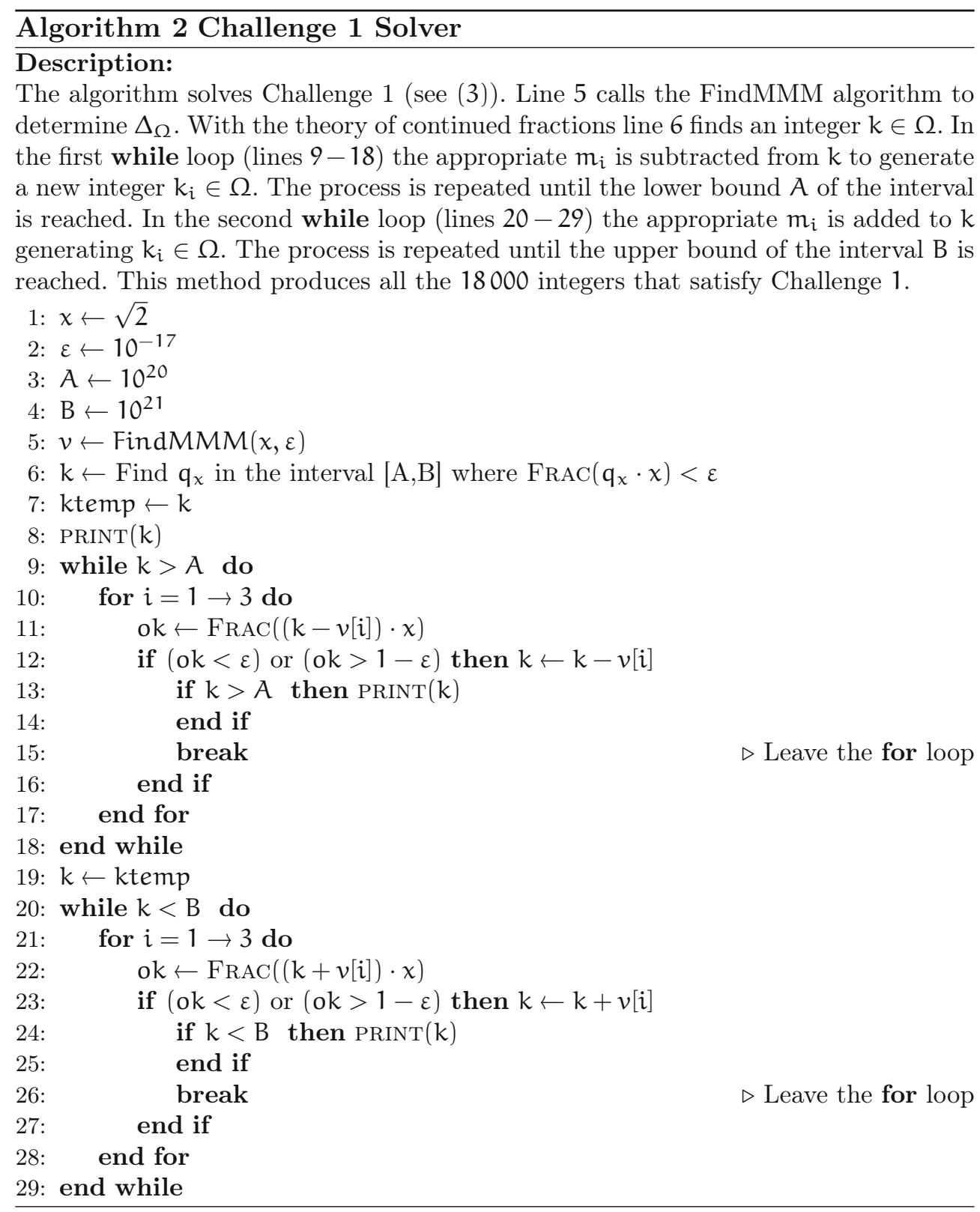




\section{2 "Many elements" approximation}

In some cases it is not necessary to find all the $k_{i}$ elements of $\Omega$, rather it is enough to find as much as possible within a given time unit. Then, the following procedure works:

Find the smallest integer $x$ that satisfies $0<\langle x\rangle<\varepsilon$ and find the smallest integer $y$ that satisfies $-\varepsilon<\langle y\rangle<0$. Using the notations (7) it is easy to see that if $\left\langle k_{i}\right\rangle \in B$ and $\langle x\rangle \in C$ then $\left\langle k_{i}+x\right\rangle \in B \cup C$. In the same way, if $\left\langle k_{i}\right\rangle \in C$ and $\langle y\rangle \in B$ then $\left\langle k_{i}+y\right\rangle \in B \cup C$. Only with these two integers it is always possible to produce a subset of $\Omega$.

Example 5 (cont.) If we want to determine just "many" elements of $\Omega$, the previous method generates 12945 integers within 15 ms.

\section{Approximations for the multi-dimensional case}

\section{1 "Many elements" approximation}

Calculating all-elements of $\Omega$ seems to be hard in higher dimensions. However, we can generalize our one dimensional method to find "many" $q \in \Omega$ integers recursively. The algorithm is based on the following lemma:

Lemma 8 Let the irrationals $\alpha_{1}, \alpha_{2}, \ldots, \alpha_{n}$ and the real $\varepsilon>0$ be given. Then there is a set $\Gamma_{\mathrm{n}}$ with $2^{\mathrm{n}}$ elements with the following property: if $\mathrm{q} \in \Omega$ then $\mathrm{q}+\gamma \in \Omega$ for some $\gamma \in \Gamma_{\mathrm{n}}$.

Proof. Let $\mathrm{q} \in \Omega$ be given. Let us define an $\mathrm{n}$-dimensional binary vector $\mathrm{b}$ associated with $\mathrm{q}$ in the following way:

$$
b_{i}= \begin{cases}1 & \text { if } q \alpha_{i}-\left\|q \alpha_{i}\right\| \in \mathbb{N}, \\ 0 & \text { if } q \alpha_{i}+\left\|q \alpha_{i}\right\| \in \mathbb{N} .\end{cases}
$$

Let $\Gamma_{n}$ be the set for which

1. $\gamma \in \Gamma_{\mathrm{n}}$ implies $\left\|\mathrm{q} \alpha_{\mathrm{i}}\right\|<\varepsilon$ for all $1 \leq \mathrm{i} \leq \mathrm{n}$,

2. all the associated binary representations by (8) are different.

Then, for a given $\mathrm{q} \in \Omega$ there exists a $\gamma \in \Gamma_{\mathrm{n}}$ such that $\mathrm{q}+\gamma \in \Omega$, e.g. when their associated binary representations are (1's binary) complements. Clearly, $\left|\Gamma_{\mathrm{n}}\right|=2^{\mathrm{n}}$. The proof is finished. 
A. Kovács, N. Tihanyi

$\begin{array}{lllll}356205059916 & 3487229338057 & 3565485794412 & 3921690854328 & 4576624903864 \\ 5800642344603 & 7056176493393 & 7134432949748 & 7490638009664 & 9054007777845 \\ 10977867347721 & 11591199235356 & 11889764427290 & 12324225943561 & 15811455281618 \\ 16900850847425 & 17257055907341 & 18046611831809 & 18152923635291 & 18647375728749 \\ 18725632185104 & 19081837245020 & 19380402436954 & 19814863953225 & 20686645377416 \\ 20960788735295 & 21721870790627 & 22050020503416 & 22945888231366 & 23302093291282 \\ 23957027340818 & 25181044781557 & 25537249841473 & 25822432016319 & 27522513135230 \\ 27878718195146 & 28790615274715 & 29102735635885 & 29703499532825 & 31938492285330 \\ 32712306129043 & 35925048224463 & 38160204774654 & 39113548572900 & 39113712370586 \\ 40202944138707 & 41949469020031 & 42383930536302 & 42438100688898 & 44262882026577 \\ 44423200184969 & 44619087086493 & 44917652278427 & 47693582148371 & 51437938314147 \\ 51794143374063 & 52092708565997 & 56669333469861 & 58494114807540 & 59583346575661 \\ 60430542368111 & 62415805661868 & 62714370853802 & 63070575913718 & 65007167271975 \\ 65305732463909 & 65383988920264 & 66992266768046 & 67564975317859 & 68559097897151 \\ 68871218258321 & 75394965654965 & 76540726639349 & 76718061327901 & 76975188155620 \\ 79615221701227 & 79971426761143 & 81850378251418 & 82152413158738 & 84031364649013 \\ 84387569708929 & 87953055503341 & 88607825755191 & 88686082211546 & 91522002658677 \\ 91562625996499 & 92173311549603 & 95131573151835 & 95443693513005 & 96098463764855 \\ 98618802489892 & 98697058946247 & 100932215496438 & 103273520052425 & 104419444834495 \\ 105152471542700 & 107689663000211 & 112821979923728 & 119085139131729 & 121320131884234 \\ 140045764069338 & 140401969129254 & 143970916284590 & 147101940562731 & 151379836476975 \\ 153024924062435 & 156512153400492 & 161661323056483 & 164002791410156 & 170838495370284 \\ 175415120274148 & 179814246691774 & 183383193847110 & 189974502767900 & 204205735548863 \\ 208621878496649 & 208998700144938 & 261026707423816 & 266621541210731 & 269101092800260 \\ 269457297860176 & 299828135635546 & 305300628267360 & 320949406326919 & 331272339625104 \\ 382947603204990 & 408250989141648 & 616256074389738 & & \end{array}$

Table 1: The result of the precalculation for solving Challenge 2

Remark 9 Computing the appropriate $\gamma \in \Gamma_{\mathrm{n}}$ for a given $\mathrm{q} \in \Omega$ is not necessarily unique.

Corollary 10 Remember the first dimension case: For all $\mathrm{m} \in \mathbb{N}, \mathrm{q} \in \Omega$, $\langle\mathrm{q}+\mathrm{m}\rangle \in \mathrm{B} \cup \mathrm{C}$ implies that $\langle\mathrm{m}\rangle \in \mathrm{A} \cup \mathrm{B} \cup \mathrm{C} \cup \mathrm{D}$. We can generalize this to higher dimensions. Let $\mathrm{q} \in \Omega$ and $\mathrm{m} \in \mathbb{N}$ be given. Then $\mathrm{q}+\mathrm{m} \in \Omega$ implies $\left\|\mathrm{m} \cdot \alpha_{\mathrm{i}}\right\| \in \mathrm{A} \cup \mathrm{B} \cup \mathrm{C} \cup \mathrm{D}$ for all $1 \leq \mathrm{i} \leq \mathrm{n}$.

Unfortunately, the precalculation of the $2^{\mathfrak{n}}$ integers is in general computationally expensive. However, there are several tricks based upon Lemma 8 that can be applied to make the generation more efficient.

Example 5 (cont.) In Challenge 2 the precalculation of the $2^{7}=128$ integers took approximately 6.14 sec on our architecture. Table 1 shows the result. Applying the Challenge 2 Solver we were able to produce 120852 integers in $\Omega$ within 26.8 sec. 


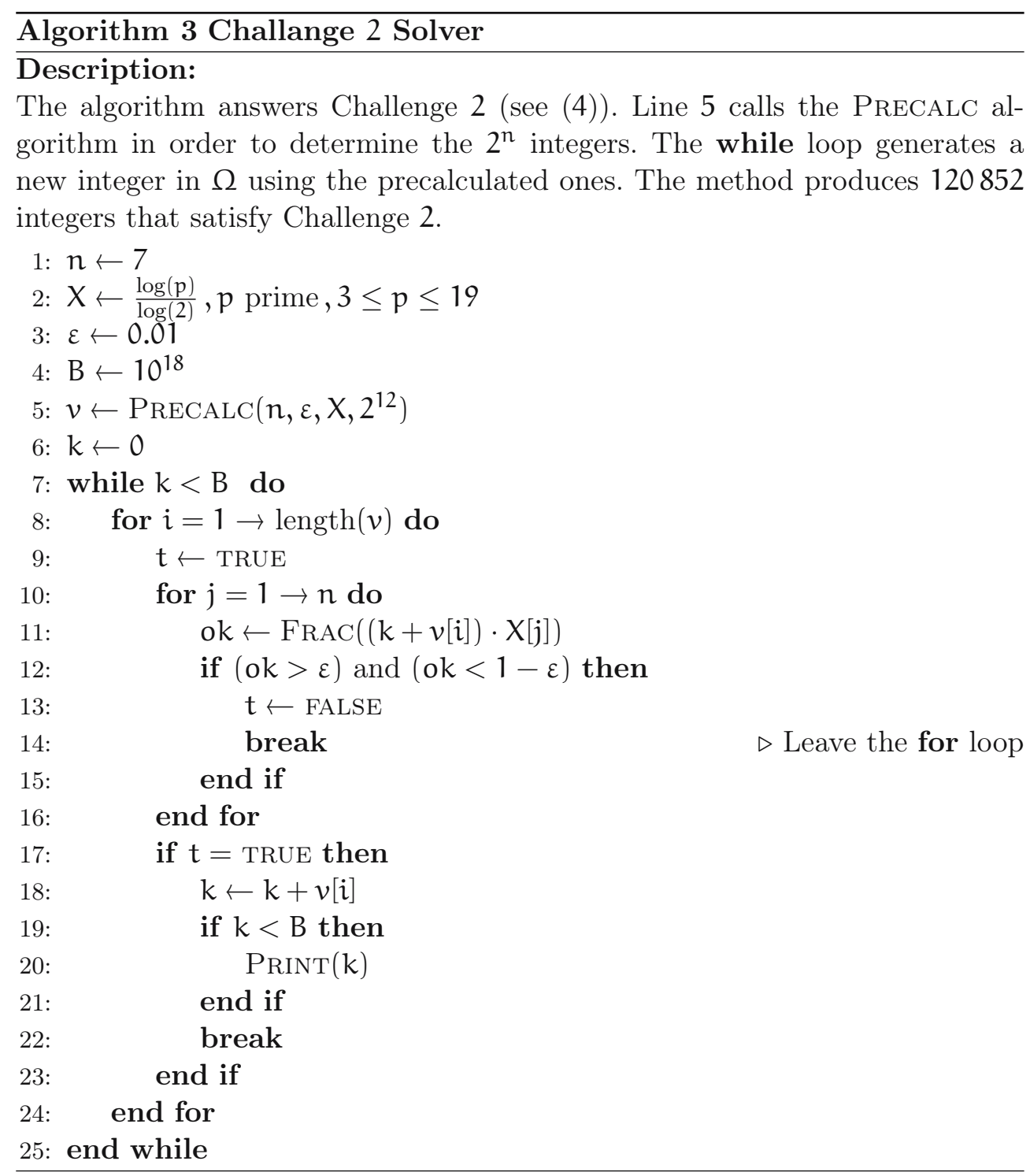


A. Kovács, N. Tihanyi

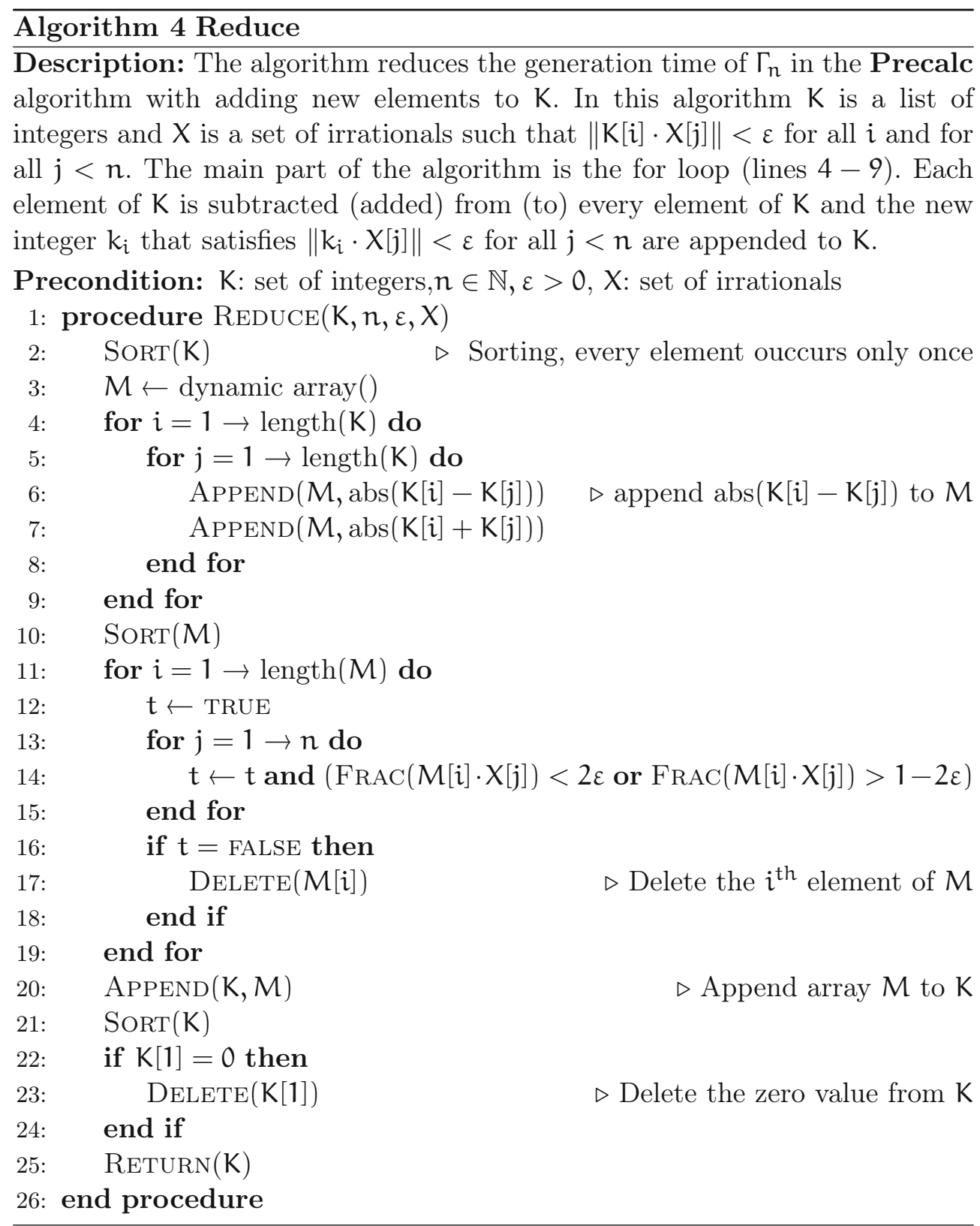




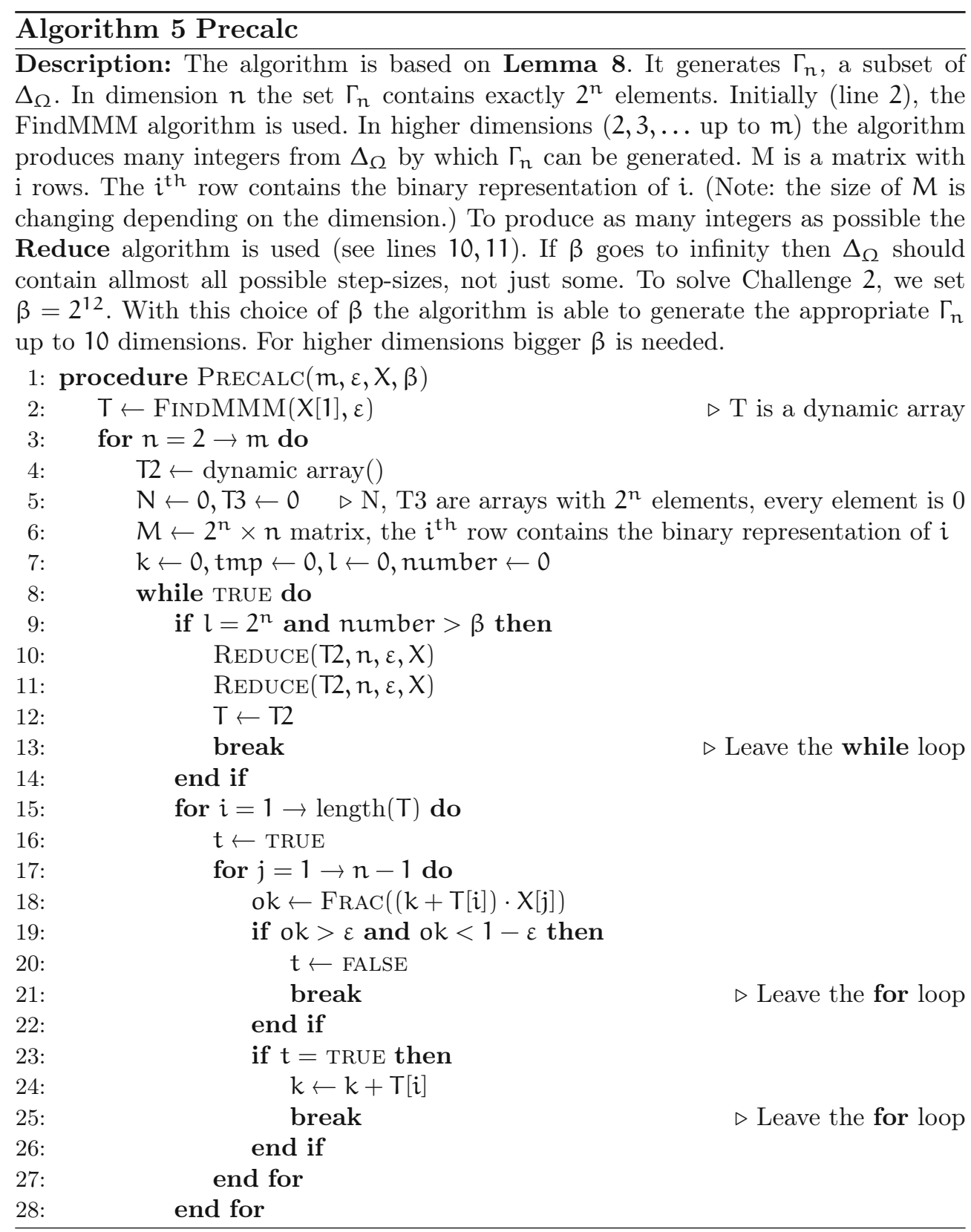




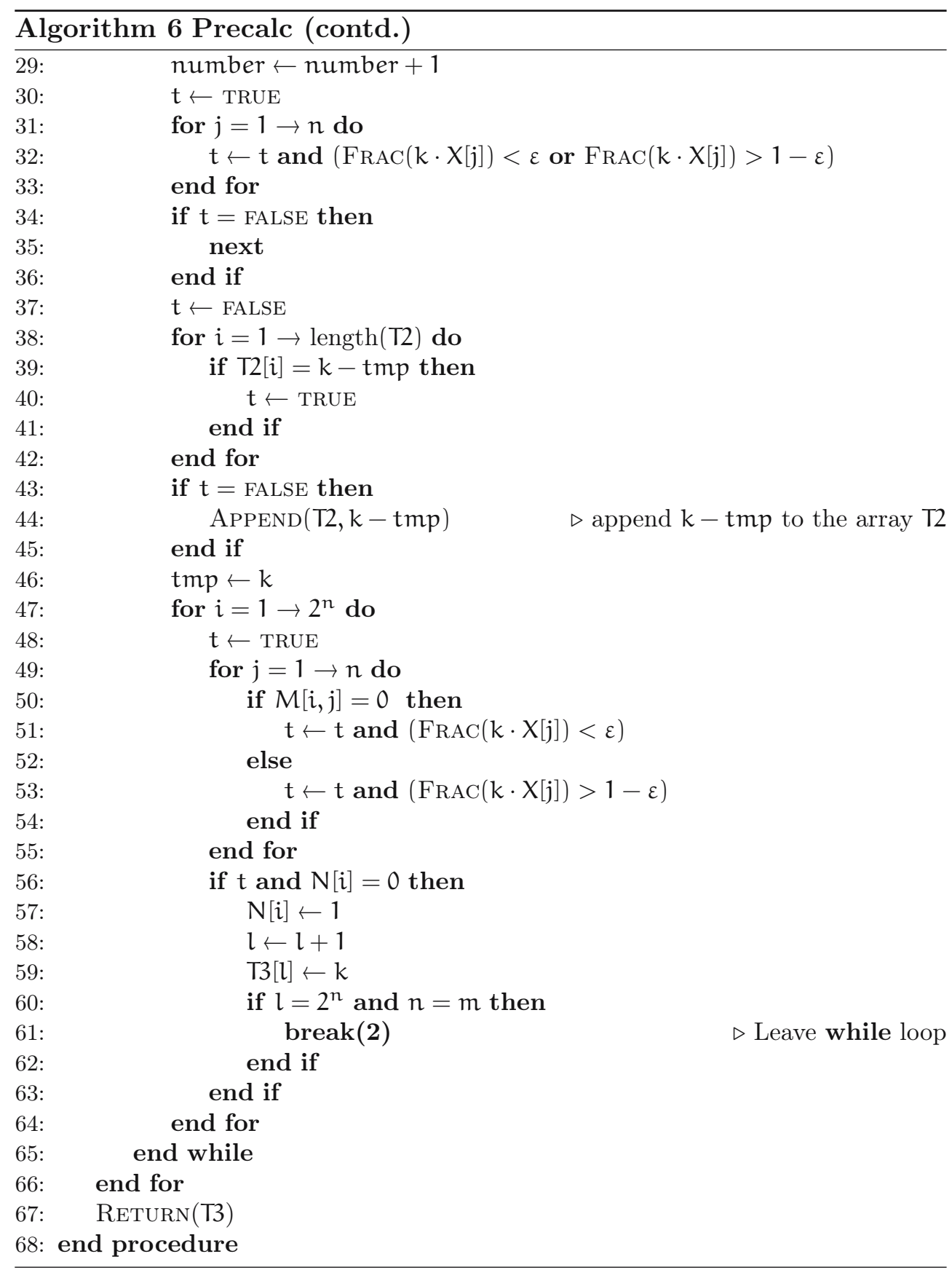




\section{Practical use of our methods}

The real power of the presented methods is the ability to use them in a distributed way.

There are several fields of mathematics where the techniques shown in this paper can be applied. We used our methods in order to find high peak values of the Riemann-zeta function effectively. It is computationaly hard to find real $t$ values where $|\zeta(1 / 2+i t)|$ is high (see [11]). In 2004 Tadej Kotnik observed that large values of $|\zeta(1 / 2+i t)|$ are expected when $t=\frac{2 k \pi}{\log 2}$, where $k \frac{\log \left(p_{i}\right)}{\log (2)}$ are close to an integer for all primes $p_{i}>2$ [6]. The methods shown in this paper can be used to find thousands of candidates within a few minutes where high values of $|\zeta(1 / 2+i t)|$ are expected. We plan to continue our research in this direction.

\section{Acknowledgement}

The authors would like to thank Prof. Dr. Antal Járai for his very helpful comments, suggestions and to the anonymous reviewers for many constructive comments. The research of the first author was partially supported by the European Union and co-financed by the European Social Fund (ELTE TÁMOP-4.2.2/B-10/1-2010-0030).

\section{References}

[1] F. Armknecht, C. Elsner, M. Schmidt, Using the Inhomogeneous Simultaneous Approximation Problem for Cryptographic Design. AFRICACRYPT, 2011, pp. $242-259 . \Rightarrow 18$

[2] A. Frank, É. Tardos, An application of simultaneous Diophantine approximation in combinatorial optimization, Combinatorica, 7, 1 (1987) 49-66. $\Rightarrow 18$

[3] A. Y. Khinchin, Continued Fractions, Translated from the third (1961) Russian edition, Reprint of the 1964 translation, Dover, Mineola, NY, 1997. $\Rightarrow 20$

[4] Sh. Kim, S. Östlund, Simultaneous rational approximations in the study of dynamical systems, Phys. Rev. A, 34, 4 (1986) 3426-3434. $\Rightarrow 18$

[5] C. Kimberling, Best lower and upper approximates to irrational numbers, Elem. Math., 52, 3 (1997) 122-126. $\Rightarrow 20$

[6] T. Kotnik, Computational Estimation of the order of $\zeta(1 / 2+i t)$, Math. Comp., 73, 246 (2004) 949-956. $\Rightarrow 33$

[7] J. C. Lagarias, Best simultaneous Diophantine approximations I., Growth rates of best approximation denominators, Trans. Am. Math. Soc., 272, 2 (1982) 545554. $\Rightarrow 18$ 
[8] J. C. Lagarias, Best simultaneous Diophantine approximations II., Behavior of consecutive best approximations, Pacific J. Math., 102, 1 (1982) 61-88. $\Rightarrow 18$

[9] J. C. Lagarias, The computational complexity of simultaneous Diophantine approximation problems, SIAM J. Computing 14, 1 (1985) 196-209. $\Rightarrow 18$

[10] A. K. Lenstra, H. W. Lenstra Jr., L. Lovász, Factoring polynomials with rational coefficients, Math. Ann., 261, 4 (1982) 515-534. $\Rightarrow 18,21$

[11] A. M. Odlyzko, The $10^{20}$-th zero of the Riemann zeta function and 175 million of its neighbors, 1992 (unpublished) $\Rightarrow 33$

[12] V. T. Sós, G. Szekeres, Rational approximation vectors, Acta Arithm., 49, 3 (1988) $255-261 . \Rightarrow 18$

Received: April 10, 2013•Revised: June 8, 2013 